\title{
Nanog and transcriptional networks in embryonic stem cell pluripotency
}

\author{
Guangjin Pan ${ }^{1}$, James A Thomson ${ }^{1,2,3,4}$
}

${ }^{1}$ The Wisconsin National Primate Research Center, University of Wisconsin-Madison, 1220 Capitol Court, Madison, WI 53715-1299, USA; ${ }^{2}$ The Genome Center of Wisconsin, 425 Henry Mall, Madison, WI 53706, USA; ${ }^{3}$ WiCell Research Institute, PO Box 7365 , Madison, WI 53707-7365, USA; ${ }^{4}$ The Department of Anatomy, University of Wisconsin-Madison Medical School, 470 N. Charter Street, Madison, WI 53706-1509, USA

Several extrinsic signals such as LIF, BMP and Wnt can support the self-renewal and pluripotency of embryonic stem (ES) cells through regulating the "pluripotent genes." A unique homeobox transcription factor, Nanog, is one of the key downstream effectors of these signals. Elevated level of Nanog can maintain the mouse ES cell self-renewal independent of LIF and enable human ES cell growth without feeder cells. In addition to the external signal pathways, intrinsic transcription factors such as FoxD3, P53 and Oct4 are also involved in regulating the expression of Nanog. Functionally, Nanog works together with other key pluripotent factors such as Oct4 and Sox 2 to control a set of target genes that have important functions in ES cell pluripotency. These key factors form a regulatory network to support or limit each other's expression level, which maintains the properties of ES cells.

Cell Research (2007) 17:42-49. doi:10.1038/sj.cr.7310125; published online 9 January 2007

Keywords: Nanog, embryonic stem cell pluripotency, transcription factor

\section{Introduction}

Derived from the inner cell mass (ICM) of the blastocyst, embryonic stem (ES) cells can proliferate indefinitely in vitro (self-renewal) and can differentiate into cells of all three germ layers (pluripotency). These unique properties make them exceptionally valuable for cell replacement therapies, drug discovery and regenerative medicine. The ES cell era began in the early 1980s when mouse ES cells were successfully derived $[1,2]$. These cells can re-enter the developing embryo and contribute to all cell types of the embryo including germ line, thus making complex genetic modifications of mice possible. More recently, the successful derivation and propagation of human ES cells has gained a great deal of attention for their potential in regenerative medicine and in the study of early human

Correspondence: Guangjin Pan

E-mail: gpan@primate.wisc.edu development [3].

Why can ES cells self-renew indefinitely without loss of pluripotency? Although ES cell research began more than 20 years ago, still little is known about the mechanisms of this unique ability. Several extrinsic growth factors support the pluripotency of ES cells. In vitro, leukaemia inhibitory factor (LIF) supports the undifferentiated state of mouse ES cells by activating the signal transducer STAT3 [4]. LIF withdrawal or direct inhibition of STAT3 causes mouse ES cells to differentiate into a morphologically mixed cell population [5]. However, LIF receptor gp130-/- mouse embryo can develop to a stage beyond that of ES cell derivation, suggesting that additional factors may also be involved in maintaining pluripotent cells in vivo [6]. Another extrinsic factor known to support mouse ES cell self-renewal is BMP4. In the presence of LIF, BMP4 can enhance the self-renewal and pluripotency of mouse ES cells by activating members of the $I d$ gene (inhibition of differentiation) family [7]. In addition to LIF and BMP4, Wnt pathway is shown to delay the onset of differentiation of mouse and human ES cells in short-term experiments 
[8]. However, the role of Wnt/beta-catenin signaling in ES cell self-renewal is a debatable issue as different models have been proposed by others [9]. Interestingly, under conditions that would promote self-renewal of mouse ES cells, LIF is not sufficient to maintain human ES cells and BMPs cause rapid differentiation [10, 11]. Instead, FGF signaling and a balance between TGF $\beta /$ Activin and BMP signaling appear central to the self-renewal of human ES cells [12-14].

Functionally, external signaling eventually leads to distinct regulation of genes that result in the pluripotent state. The best-characterized gene of these is Oct4, which functions to maintain pluripotency both in vivo and in vitro (for a review see [15]). Oct4 is a POU domain transcription factor that is specifically expressed in all pluripotent cells during mouse embryogenesis and also in undifferentiated ES cells. Loss of pluripotency in ES cells is often accompanied by Oct4 downregulation. Oct4 deficient mouse embryos fail to develop beyond the blastocyst stage due to the lack of pluripotent ICM [16], suggesting the critical role of Oct4 in maintaining ES cell pluripotency in vivo. However, Oct4 is not the only master gene that controls ES cell pluripotency. On LIF withdrawal, Oct4 on its own is not sufficient to prevent mouse ES cell differentiation, suggesting that additional new factors are also required. About 3 years ago, another "master gene" in pluripotency, Nanog, was discovered.

\section{Nanog, a new "master gene" of ES cell pluripo- tency}

\section{Identification of Nanog}

A more recently described gene, Nanog, plays a critical role in regulating the cell fate of the pluripotent ICM during embryonic development, maintaining the pluripotent epiblast and preventing differentiation to primitive endoderm [17]. By using PCR to screen a mouse ES cell cDNA library, Wang et al. [18] described an ENK gene (early embryo specific NK) specifically expressed in ES cells. This gene has a homeodomain with homology to members of the NK gene family, but its function was unknown at that time. This gene was later re-cloned and re-named as Nanog by two independent groups that analyzed its function. Screening for critical factors that can maintain ES cell pluripotency independently of the LIF-STAT3 pathway, Chambers et al.[17] and Mitsui et al.[19] identified the same transcription factor, though using different strategies , and named it as Nanog. Nanog mRNA is first detected in the interior cells of the compacted morulae during mouse embryo development, then confined to the ICM, and disappears in the trophectoderm in the blastocyst stage. In later blastocysts, Nanog expression is further restricted in the epiblast and excluded from the primitive endoderm. After implantation, Nanog expression is downregulated, but can be detected in germ cells of the genital ridges of E11.5 mouse embryos [17]. In vitro, Nanog mRNA is enriched in pluripotent cell lines such as ES, EG and EC (embryonic carcinoma) cells, but not in adult tissues [17]. On differentiation of these pluripotent cells, Nanog expression is downregulated. Mouse embryos lacking Nanog fail to develop beyond the blastocyst stage due to the absence of epiblasts [19]. Nanog-/- ES cells can be derived from the Nanog-/- blastocyst, but display slow differentiation to extra embryonic endoderm in vitro [19]. For mouse ES cells, the most prominent role of Nanog is that a high level of Nanog can maintain pluripotency in the absence of extrinsic factor LIF, suggesting that Nanog may be a major downstream effector for extrinsic factors. More recently, research on human ES cells demonstrates that high levels of Nanog in human ES cells enable them to grow well in a feeder-free condition [20]. Further microarray data suggest that these cells display epiblast properties, suggesting that Nanog is beneficial to the maintenance of epiblast. In contrast, downregulation of Nanog can induce both mouse and human ES cell differentiation to extra-embryonic lineages $[21,22]$. Thus, although human and mouse ES cells show significant differences in their morphology, cell surface markers and growth factor dependence, a central role of Nanog appears to be conserved.

\section{Nanog is a unique homeobox transcription factor}

Mouse Nanog protein consists of 280 amino acids and contains a homeobox domain. This homeodomain shows some homology to that of the NK2 gene family, but the amino acids identity is less than $50 \%$. In addition to its ortholog in human, rat, mouse, dog and chimpanzee, phylogenic analysis did not reveal other homologous proteins, suggesting that it appears to be a unique homeobox protein. Structurally, Nanog can be considered simply as a threedomain protein - N-terminal domain, homeodomain and C-terminal domain. $\mathrm{N}$-terminal domain contains 96 amino acids with serine rich, and C-terminal domain contains a unique and well-conserved 50 amino acids tryptophan repeat domain (Figure 1). When fused to the DNA-binding domain of Gal4, both $\mathrm{N}$ - and C-terminal domains show trans-activator function [23], but the activity of the C-terminal domain is much higher (at least seven times) than the N-terminal domain [23]. Similar analysis on human Nanog protein reveals that only the C-terminal domain has transcriptional activity [24], suggesting that the C-terminal domain is functionally dominant. The prominent feature of the C-terminal domain is the presence of a 10 pentapeptide repeat (WR), each starting with a tryptophan (W). This repeat is well conserved between human and mouse, 

$\mathrm{N}$ domain

Rattus norvegic Mus musculus Canis familiari Pan troglodytes Homo sapiens

Rattus norvegic Mus musculus Canis familiari Pan troglodytes Homo sapiens

Rattus norvegic Mus musculus Canis familiari Pan troglodytes Homo sapiens

Rattus norvegic Mus musculus Canis familiari Pan troglodytes Homo sapiens

Rattus norvegic Mus musculus
1

1

1

1

1

MSVDLSGPHSLPSCEEASNSGDSSPMPAVHLPEENYSCLQVSATEMLCTETASPPPSSGD MPAVFHP-ENYSCLQGSATEMLCTEAASPRPSSED MPAGPQAPNSR----------DPSPMPEVYGPRGNPASLPMSSAETPHAETVSPLPSSMD MSVDPACPQSLP-CFEASDCKESSPMPVICGPEENYPSLQMSSAEMPHTETVSPLPSSMD MSVDPACPQSLP-CFEASDCKESSPMPVICGPEENYPSLQMSSAEMPHTETVSPLPSSMD

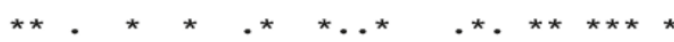

61 LPLQDSPDSSSNPKLKLSGPEADEGPEKKEENKVLTKKQKMRTVFSQAQLCALKDRFQRQ 35 LPLQGSPDSSTSPKQKLSSPEADKGPEE-EENKVLARKQKMRTVFSQAQLCALKDRFQKQ 51 LLTQDSPDSSTSPRVKLPPTSGEERTAR-KEDATQGKKQKMRTVFSQTQLYVLNDRFQRQ 60 LLIQDSPDSSTSPKGKQP-TSAENSVTK-KEDKVPVKKQKTRTVFSSTQLCVLNDRFQRQ 60 LLIQDSPDSSTSPKGKQP-TSAENSVAK-KEDKVPVKKQKTRTVFSSTQLCVLNDRFQRQ

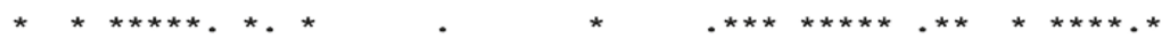

121 RYLSLQQMQDLSTILNLSYKQVKTWFQNQRMKCKRWQKNQWLKTSNGLTQKGSAPVEYPS 94 KYLSLQQMQELSSILNLSYKQVKTWFQNQRMKCKRWQKNQWLKTSNGLIQKGSAPVEYPS 110 KYLSLQQMQELSNILNLSYKQVKTWFQNQRMKSKRWQKSNWPKESNSVTQN-SSATTEYA 118 KYLSLQQMQELSNILNLSYKQVKTWFQNQRMKSKRWQKNNWPKNSNGVTQK-ASAPTYPS 118 KYLSLQQMQELSNILNLSYKQVKTWFQNQRMKSKRWQKNNWPKNSNGVTQK-ASAPTYPS

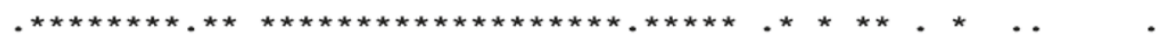
181 IHCSYSQGYLMNASGNLPVWGSQTWTNPTWNNQTWTNPTWSNQTWTNPTWSNQAWSTQSW 154 IHCSYPQGYLVNASGSLSMWGSQTWTNPTWSSQTWTNPTWNNQTWTNPTWSSQAWTAQS169 GFYPCRQGYLLNPSGNLPLWSSQAWNNPNWSSQTWNSQSWSSHSWNSQTWCPQAWNNQAW 177 LYSSYHQGCLVNPTGNLPMWSNQTWNNSTWSNQTQNIQSWSNHSWNTQTWCTQSWNNQAW 177 LYSSYHQGCLVNPTGNLPMWSNQTWNNSTWSNQTQNIQSWSNHSWNTQTWCTQSWNNQAW

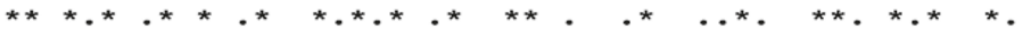
241 CTQAWNSQTWNAAPLHNFGEDSLQPYVPLQQNFSASDLEANLEATRESQ-------AHFS 213 ----WNGQPWNAAPLHNFGEDFLQPYVQLQQNFSASDLEVNLEATRESH-------AHFS 229 N-----------NPLHNCEEESLQPPIQFQQNS-MGDLES IFETAGESHGVLQQSTKYFS 237 N----------SPFYNCGEESLQSCMQFQPNSPASDLEAALEAAGEGLNVIQQTARYFS 237 N-----------SPFYNCGEESLQSCMQFQPNSPASDLEAALEAAGEGLNVIQQTTRYFS

294 TPQALELFLNYSVNSPGEI 312

262 TPQALELFLNYSVTPPGEI 280

277 TPQIMDFFPNYSEHST 292

286 TPQTMDLFLNYSTNMQPEDM 305

286 TPQTMDLFLNYSMNMQPEDV 305

$\star \star \star \ldots * \star \star * *$

Figure 1 Schematic structure of Nanog. The alignment of full-length Nanog from rat, mouse, dog, chimpanzee and human species was generated by Clustal W. The functional domains are indicated as different colors.

although one of the tryptophans is replaced by a glutamine (Q) in human Nanog. Functional analysis shows that WR works as a strong trans-activator and the identity of $\mathrm{W}$ is critical to its function [25]. Replacement of W with A(Ala) completely abolishes its activity. In addition to WR, the
C-terminal domain also contains another trans-activator module, the 58 amino acids region $\mathrm{C}$-terminal to the WR (CD2) [25]. Both WR and CD2 can separately trans-activate the expression of a reporter gene [25]. Thus, WR and $\mathrm{CD} 2$ seem to be functionally redundant in the regulation 
of target genes, but given the structural differences, it will be interesting to know whether these two domains function differently when Nanog binds to endogenous target genes in vivo.

\section{Combinatorial signaling of Nanog with other fac- tors}

\section{Nanog and external signals}

In vitro, LIF and activated STAT3 are able to maintain the undifferentiated state in mouse ES cells. When a conditionally active form of STAT3 is induced by tamoxifen, the phenotype of mouse ES cells can be maintained in the absence of LIF [26]. Similarly, if Nanog is overexpressed, mouse ES cells also do not need LIF to maintain its pluripotency. If Nanog is overexpressed in mouse ES cells, the level of phosphorylated STAT3 does not change significantly compared with wild type, and elevated STAT3 signaling seems not to affect Nanog expression [17]. These findings suggest that Nanog is not a direct transcriptional target of STAT3, nor does Nanog regulate STAT3. More recently, however, analysis of the mouse Nanog 5 ' promoter region reveals a STAT3 binding site $5 \mathrm{~kb}$ upstream of the translation start site [27]. A putative $\mathrm{T}$ (Brachyury) binding site is also found in this region [27]. Chromatin immunoprecipitation (Chip) assay shows that STAT3 and T can indeed bind to this region in vivo. A luciferase reporter gene, driven by the $5.2 \mathrm{~kb}$ of Nanog 5 ' promoter region containing these two binding sites, can be significantly upregulated by LIF. According to these recent findings, Nanog may be a direct downstream effector of the LIF-STAT3 pathway in maintaining ES cell pluripotency, which is consistent with the fact that high levels of Nanog can bypass the requirement for LIF to maintain the undifferentiated state of mouse ES cells. However, this remains the only report suggesting that Nanog is a direct target of the LIF-Stat3 pathway. More studies are needed to confirm the direct link between Nanog and LIF-Stat3 signaling.

Bone morphogenetic protein (BMP) signaling functions in mesoderm-induction during embryonic development [28], but it seems to have different effects on pluripotent ES cells. In the absence of LIF, low concentrations of BMPs promote mesoderm differentiation of mouse ES cells, while in the presence of LIF the same concentration of BMPs maintains ES cell pluripotency [7]. BMPs belong to the TGFb super family and mediate signaling through their downstream effectors - SMAD1 or the closely related SMAD5 and SMAD8. Interestingly, it was found that Nanog can physically interact with SMAD1 and interfere with the further recruitment of the coactivators to the active SMAD1 complexes, thus inhibiting the activity of BMP signaling [27]. Combined with the role of BMPs on ES cell pluripotency and differentiation, a negative feedback mechanism was proposed on the relationship between Nanog and BMPs [27]. In this model, BMPs initially promote mesoderm differentiation with upregulation of the mesoderm marker-Brachyury and prevent the neural-ectoderm differentiation. In the presence of LIF, activated STAT3 interacts with Brachyury and binds the Nanog promoter (Figure 2), resulting in upregulation of Nanog expression. Then, elevated levels of Nanog, in turn, block the activity of BMPs by interfering with their effector-SMAD1, thus limiting mesoderm progression and ultimately maintaining the undifferentiated state of mouse ES cells.

\section{Regulation of Nanog}

Nanog expression is restricted to pluripotent cells and is downregulated upon differentiation, but little is known about how Nanog expression is regulated. Through analysis of its 5' promoter region, a composite Oct4/Sox 2 motif localized -180 bp upstream of the transcription start site was found to be important for Nanog regulation (Figure 2) [29, 30]. A reporter gene driven by the Nanog proximal promoter containing the Oct $4 / \mathrm{Sox} 2$ motif recapitulates appropriate Nanog expression in pluripotent and non-pluripotent cells, and this motif is well-conserved between mouse, rat and human [30]. Electrophoretic mobility shift and Chip assays demonstrate that Oct4 and Sox 2 indeed bind to the Nanog promoter in vitro and in vivo [29]. Further mutagenesis analysis of this site shows that Oct4/Sox 2 motif is required for the activity of Nanog promoter in pluripotent cells [30]. These findings suggest that Oct4/Sox act to promote Nanog transcription in pluripotent cells. However, high levels of Nanog are beneficial to ES cell self-renewal, but overexpressed Oct4 induces differentiation. Moreover, in Oct4 deficient embryos, Nanog expression can be readily detected by mRNA in situ hybridization [17], suggesting that Nanog can be maintained without Oct4. These findings indicate that other pluripotent factors may contribute to the regulation of Nanog expression. FoxD3, a forkhead family transcription factor, is highly expressed in mouse ES cells and in pluripotent cells of the early embryo [31]. FoxD3 null mouse embryos die shortly after implantation due to the loss of the epiblast, a phenotype somewhat similar to the Nanog deficient embryos [32], and FoxD3 is an activator of Nanog [33]. Reporter assays show that FoxD3 can activate the Nanog promoter through an ES cell-specific enhancer localized at -270 upstream of the transcription start site (Figure 2) [33]. Chip assays further demonstrate that FoxD3 indeed binds to the Nanog promoter region in vivo [33]. FoxD3 was originally known as a transcription repressor, but can nevertheless activate the Nanog transcription, suggesting that gene context may determine the outcome of FoxD3 regulation. 


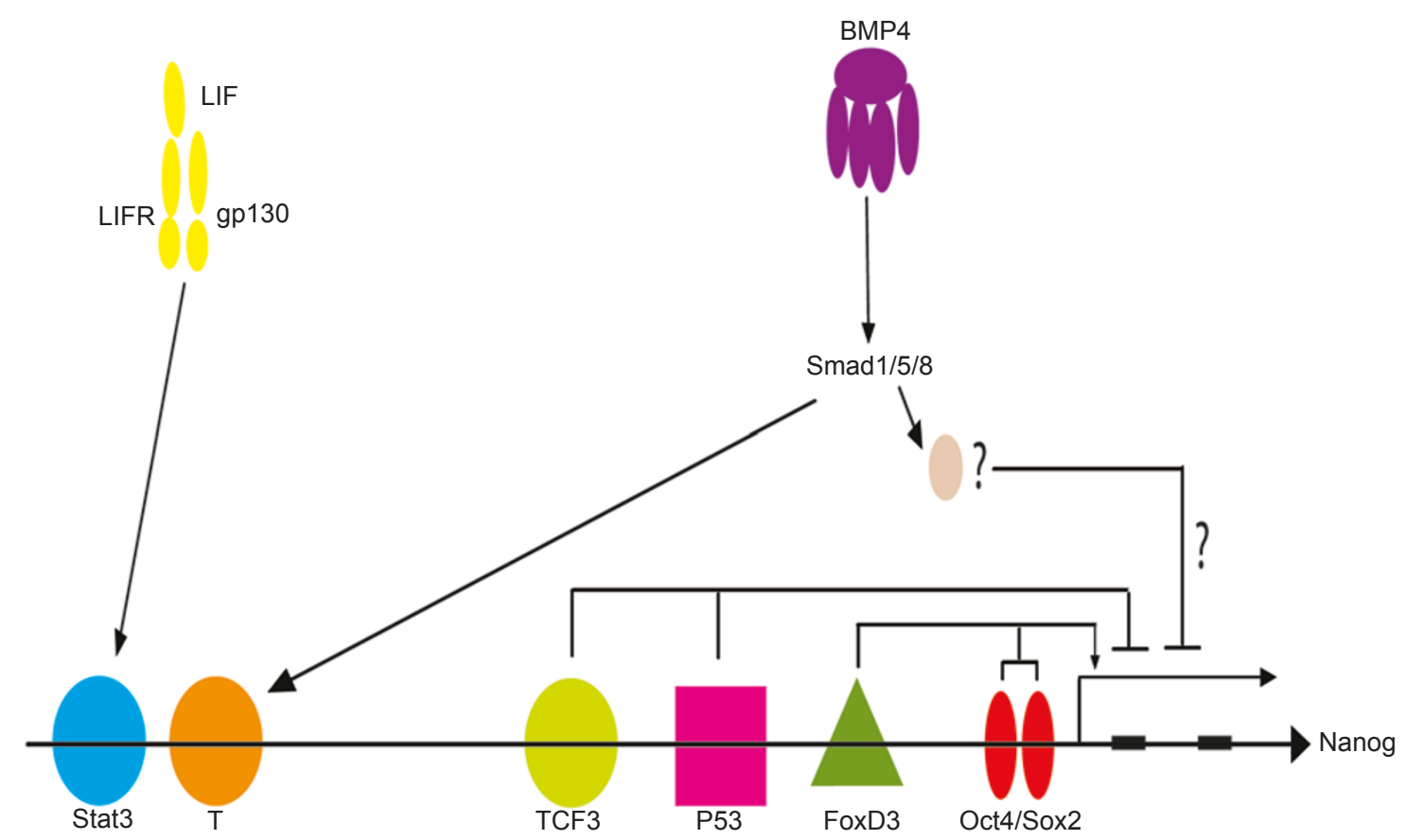

Figure 2 Regulation of Nanog expression. FoxD3 and Oct4/Sox2 bind to the proximal region of Nanog promoter and support its expression. TCF3 and p53 also bind to the promoter and negatively regulate Nanog expression. LIF and BMP signaling and their downstream effectors STAT3 and T may also be involved in Nanog regulation.

Functionally, Nanog blocks differentiation. Thus, negative regulation of Nanog is required to promote differentiation during embryonic development. The tumor suppressor p53 can bind to the Nanog promoter as suggested in vitro and in vivo evidence (Figure 2) [34]. During ES cell differentiation, the rapid downregulation of Nanog correlates with the induction of the transcription activity and Ser315 phosphorylation of p53 [34]. Impairing the induction of p53 activity by knock-in of $\mathrm{p} 53^{\mathrm{S} 315 \mathrm{~A}}$ results in inefficient Nanog suppression during ES cell differentiation. These findings suggest that p53 may be one of the negative regulators of Nanog during ES cell differentiation. However, when p53-/- ES cells are treated with retinoic acid (RA), Nanog is still downregulated during the differentiation [34]. Therefore, there must be other negative factors involved in suppressing Nanog during ES cell differentiation. Tcf3, a transcription factor that functions downstream of the Wnt pathway, is highly expressed in undifferentiated mouse ES cells. Ablation of Tcf3 in mouse ES cells leads to delayed differentiation in favor of self-renewal with elevated levels of Nanog protein [35]. Further Chip and reporter assays showed that Tcf3 binds to a regulatory region on the Nanog promoter and represses the promoter activity in ES cells (Figure 2) [35].

The regulatory mechanisms of Nanog expression are summarized in Figure 2. The common features that define stem cells are the ability to self-renew, and the ability to form other cell types. Thus, the levels of key factors that maintain pluripotency need to be strictly controlled to balance the maintenance of undifferentiated state and the ability of lineage commitment. These factors together form a regulatory network to support or limit each other's level, which maintains the property of ES cells (Figure 3).

\section{Transcriptional network of key pluripotent factors in ES cells}

Up to now, several transcription factors have been identified to be essential to the ES cell identity, including two principle homeodomain proteins, Nanog and Oct4, and an HMG factor, Sox2. Through chromatin immunoprecipitation (ChIP) coupled with DNA microarrays (Chips, so called CHIP-Chip), Boyer et al. [36] identified DNA regions bound individually by Oct 4 , Nanog and Sox 2 in human ES cells. The most provocative finding in this work is the high frequency of co-occupancy within the same gene region by these three factors. Among Oct4 bound genes, half of them are also bound by Sox2. Moreover, $>90 \%$ of the promoter region bound by Oct 4 and Sox 2 are also bound by Nanog. In total, 352 genes are bound by Oct4, Nanog and Sox2 simultaneously in undifferentiated 


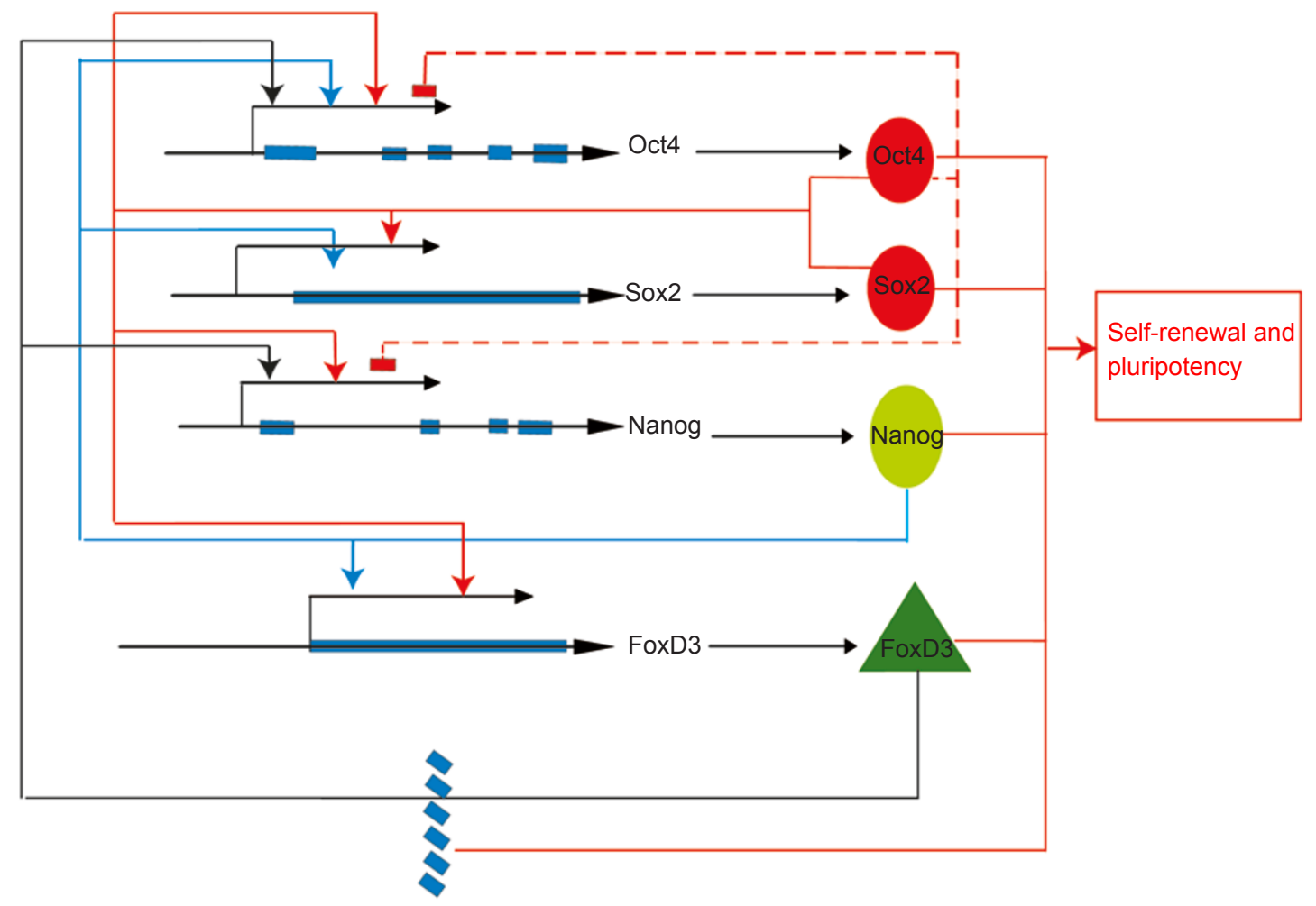

Figure 3 Regulatory network of key transcription factors in maintaining ES cell pluripotency and self-renewal. Regulators such as Oct4, Nanog, Sox 2 and FoxD3 bind to each other's promoter, and support or limit each other's expression, forming an interconnected autoregulatory network to maintain ES cell pluripotency and self-renewal. Arrows connected to factors by solid lines indicate positive regulation of a promoter by the factors. Broken lines linking to Oct4 indicate negative regulation.

human ES cells. Furthermore, their results show that Oct4, Sox 2 and Nanog are also bound to their own promoters, thus forming an interconnected autoregulation loop to maintain the ES cell identity. Indeed, this mechanism was further confirmed by data obtained through classical experimental approaches. In a recently published paper in the FASEB journal, Pan et al. [33] describe a negative feedback loop formed by Nanog, Oct4 and another pluripotent factor, FoxD3. First, Oct4 maintains Nanog expression by directly binding to a Nanog promoter when present at a sub-steady level, but represses it when Oct4 is above the normal level. On the other hand, FoxD3 positively regulates Nanog to counter the repression effect of excess Oct4 (Figure 3). Conversely, Nanog and FoxD3 function as activators for Oct 4 expression (Figure 3). When the expression level of Oct 4 rises above a steady level, it represses its own promoter as well as Nanog (Figure 3), thus exerting a negative feedback regulation loop to limit its own expression [33]. This negative feedback regulation loop keeps the expression of Oct4 at a steady level, thus maintaining the ES cell properties. Recently, another group also mapped the binding sites of Nanog and Oct4 in mouse ES cells [37]. They identified 1083 and 3006 binding sites for Oct4 and Nanog respectively in mouse ES cells, and found a substantial portion of genes bound by both Oct4 and Nanog. Through RNA interference integrated with microarray expression profiling, they showed that the core downstream targets of Oct 4 and Nanog are related to pluripotency, self-renewal and cell fate determination, which includes FoxD3, Oct4 and Sox2 [37]. Overall, these findings suggest that the key pluripotent factors always work together, rather than individually, to control a whole set of target genes, as well as each other, to keep the properties of ES cells. During the preparation of this manuscript, Wang et al. [38] identified the proteins that physically associate with Nanog in mouse ES cells. Among Nanog-associated proteins, nuclear factors with critical roles in ES cell pluripotency (including Oct4) are highly enriched. Through further screening of the partners interacting with Nanog-associated proteins, they describe a protein interaction network dedicated to pluripotency. The corresponding genes of numerous members in this network are putative or direct targets of other members, thus also forming an inter-regulation network. 


\section{Nanog and nuclei reprogramming}

Differentiated cells can be reprogrammed to the pluripotent state through transferring the nucleus into oocytes or by fusion with ES cells $[39,40]$. The successful reprogramming of a differentiated nucleus by ES cells indicates that factors expressing in ES cells may confer the pluripotency to somatic cells. The most likely candidate factor is Nanog since it functions mainly to sustain ES cell pluripotency. It has been recently reported that increasing Nanog levels in ES cells can stimulate pluripotent gene activation from the somatic cell genome through cell fusion [41]. In fusions between ES cells and neural stem (NS) cells, overexpression of Nanog in ES cells leads to an up to 200-fold increase in ES cell-characterized hybrid colonies [41]. Moreover, Nanog can also improve the yield of hybrid colonies when ES cells fuse to more differentiated cells such as thymocytes or fibroblasts [41]. These findings suggest that Nanog plays important roles in instating pluripotency in the somatic genome in ES hybrid cells, probably through opening the expression of pluripotent genes from the somatic genome. One of the known pluripotency associated genes, rex 1 , is reported to be the direct target of Nanog. Forced expression of Nanog in P19 EC cells can increase the rex 1 level [42]. In addition, expression of Nanog in NIH3T3 cells promotes the growth rate and a transformed phenotype [43]. Expression microarray analysis indicates that a portion of the Nanog target genes in ES cells are activated in Nanog-expressed NIH3T3 cells. Together, these findings suggest that Nanog has the potential to confer pluripotency on somatic cells through activating the pluripotent genes. Nevertheless, Nanog needs to act in conjunction with additional ES cell machinery, rather than operating alone, to confer pluripotency. Recently, Takahashi et al. [44] reported that mouse fibroblasts can be induced to the pluripotent state by introducing four factors, Oct4, Sox2, c-Myc and Klf4. A little unexpectedly, Nanog was dispensable for the induction. Therefore, Nanog may act as a transcriptional organizer for the hierarchy of pluripotent gene expression after the erasure of the differentiated epigenome by other factors, rather than an illuminator for the reprogramming. Indeed, expression of Nanog in neural stem cells by an expression vector did not cause any obvious phenotype change. However, when these cells were fused to ES cells, they still generated more ES cell-characterized hybrid colonies than wild-type NS cells did [41]. In the study by Takahashi et al., introduction of four factors into fibroblasts can trigger pluripotency in the absence of Nanog, but the frequency is quite low. Moreover, these induced pluripotent cells display many differences in gene expression patterns from wild-type ES cells [44]. It will be interesting to know whether elevating the level of Nanog can help these cells re-organize their gene expression program to more closely resemble that of the ES state.

\section{Acknowledgments}

We thank Deborah Faupel for reading and editing the manuscript, and members of the Thomson Lab for their kind support.

\section{References}

1 Martin G. Isolation of a pluripotent cell line from early mouse embryos cultured in medium conditioned by teratocarcinoma stem cells. Proc Natl Acad Sci 1981; 78:7634-7638.

2 Evans MJ, Kaufman MH. Establishment in culture of pluripotential cells from mouse embryos. Nature 1981; 292:154-156.

3 Thomson JA, Itskovitz-Eldor J, Shapiro SS, et al. Embryonic stem cell lines derived from human blastocysts. Science 1998; 282:1145-1147.

4 Smith AG, Heath JK, Donaldson DD, et al. Inhibition of pluripotential embryonic stem cell differentiation by purified polypeptides. Nature 1988; 336:688-690.

5 Niwa H, Burdon T, Chambers I, Smith A. Self-renewal of pluripotent embryonic stem cells is mediated via activation of STAT3. Genes Dev 1998; 12:2048-2060.

6 Nichols J, Chambers I, Taga T, Smith A. Physiological rationale for responsiveness of mouse embryonic stem cells to gp130 cytokines. Development 2001; 128:2333-2339.

7 Ying QL, Nichols J, Chambers I, Smith A. BMP induction of Id proteins suppresses differentiation and sustains embryonic stem cell self-renewal in collaboration with STAT3. Cell 2003; 115:281-292.

8 Sato N, Meijer L, Skaltsounis L, et al. Maintenance of pluripotency in human and mouse embryonic stem cells through activation of Wnt signaling by a pharmacological GSK-3-specific inhibitor. Nat Med 2004; 10:55-63.

9 Dravid G, Ye Z, Hammond H, et al. Defining the role of Wnt/betacatenin signaling in the survival, proliferation, and self-renewal of human embryonic stem cells. Stem Cells 2005; 23:14891501 .

10 Daheron L, Opitz SL, Zaehres H, et al. LIF/STAT3 signaling fails to maintain self-renewal of human embryonic stem cells. Stem Cells 2004; 22:770-778.

$11 \mathrm{Xu}$ RH, Chen X, Li DS, et al. BMP4 initiates human embryonic stem cell differentiation to trophoblast. Nat Biotechnol 2002;20:1261-1264.

$12 \mathrm{Xu}$ RH, Peck RM, Li DS, et al. Basic FGF and suppression of BMP signaling sustain undifferentiated proliferation of human ES cells. Nat Methods 2005; 2:185-190.

13 Vallier L, Alexander M, Pedersen RA. Activin/Nodal and FGF pathways cooperate to maintain pluripotency of human embryonic stem cells. J Cell Sci 2005; 118:4495-4509.

14 James D, Levine AJ, Besser D, Hemmati-Brivanlou A. TGFbeta/activin/nodal signaling is necessary for the maintenance of pluripotency in human embryonic stem cells. Development 2005; 132:1273-1282.

15 Pan GJ, Chang ZY, Scholer HR, Pei D. Stem cell pluripotency and transcription factor Oct4. Cell Res 2002; 12:321-329. 
16 Nichols J, Zevnik B, Anastassiadis K, et al. Formation of pluripotent stem cells in the mammalian embryo depends on the POU transcription factor Oct4. Cell 1998; 95:379-391.

17 Chambers I, Colby D, Robertson M, et al. Functional expression cloning of Nanog, a pluripotency sustaining factor in embryonic stem cells. Cell 2003; 113:643-655.

18 Wang SH, Tsai MS, Chiang MF, Li H. A novel NK-type homeobox gene, ENK (early embryo specific NK), preferentially expressed in embryonic stem cells. Gene Expr Patterns 2003; 3:99-103.

19 Mitsui K, Tokuzawa Y, Itoh H, et al. The homeoprotein Nanog is required for maintenance of pluripotency in mouse epiblast and ES cells. Cell 2003; 113:631-642.

20 Darr H, Mayshar Y, Benvenisty N. Overexpression of NANOG in human ES cells enables feeder-free growth while inducing primitive ectoderm features. Development 2006; 133:1193-1201.

21 Hough SR, Clements I, Welch PJ, Wiederholt KA. Differentiation of mouse embryonic stem cells after RNA interference-mediated silencing of OCT4 and Nanog. Stem Cells 2006; 24:14671475.

22 Hyslop L, Stojkovic M, Armstrong L, et al. Downregulation of NANOG induces differentiation of human embryonic stem cells to extraembryonic lineages. Stem Cells 2005; 23:1035-1043.

23 Pan GJ, Pei DQ. Identification of two distinct transactivation domains in the pluripotency sustaining factor nanog. Cell Res 2003; 13:499-502.

24 Oh J, Do H, Yang H, et al. Identification of a putative transactivation domain in human Nanog. Exp Cell Res 2005; 37:250-254.

25 Pan G, Pei D. The stem cell pluripotency factor NANOG activates transcription with two unusually potent subdomains at its C terminus. J Biol Chem 2005; 280:1401-1407.

26 Matsuda T, Nakamura T, Nakao K, et al. STAT3 activation is sufficient to maintain an undifferentiated state of mouse embryonic stem cells. EMBO J 1999; 18:4261-4269.

27 Suzuki A, Raya A, Kawakami Y, et al. Nanog binds to Smad1 and blocks bone morphogenetic protein-induced differentiation of embryonic stem cells. Proc Natl Acad Sci USA 2006; 103:10294-10299.

28 Winnier G, Blessing M, Labosky PA, Hogan BL. Bone morphogenetic protein-4 is required for mesoderm formation and patterning in the mouse. Genes Dev 1995; 9:2105-2116.

29 Rodda DJ, Chew JL, Lim LH, et al. Transcriptional regulation of nanog by OCT4 and SOX2. J Biol Chem 2005; 280:2473124737.
30 Kuroda T, Tada M, Kubota H, et al. Octamer and Sox elements are required for transcriptional cis regulation of Nanog gene expression. Mol Cell Biol 2005;25:2475-2485.

31 Sutton J, Costa R, Klug M, et al. Genesis, a winged helix transcriptional repressor with expression restricted to embryonic stem cells. J Biol Chem 1996;271:23126-23123.

32 Hanna LA, Foreman RK, Tarasenko IA, et al. Requirement for Foxd3 in maintaining pluripotent cells of the early mouse embryo. Genes Dev 2002; 16:2650-2661.

33 Pan G, Li J, Zhou Y, et al. A negative feedback loop of transcription factors that controls stem cell pluripotency and self-renewal. FASEB J 2006; 20:1730-1732.

34 Lin T, Chao C, Saito S, et al.p53 induces differentiation of mouse embryonic stem cells by suppressing Nanog expression. Nat Cell Biol 2005; 7:165-171.

35 Pereira L, Yi F, Merrill BJ. Repression of Nanog gene transcription by Tcf3 limits embryonic stem cell self-renewal. Mol Cell Biol 2006.

36 Boyer LA, Lee TI, Cole MF, et al. Core transcriptional regulatory circuitry in human embryonic stem cells. Cell 2005; 122:947956.

37 Loh YH, Wu Q, Chew JL, et al. The Oct4 and Nanog transcription network regulates pluripotency in mouse embryonic stem cells. Nat Genet 2006; 38:431-440.

38 Wang J, Rao S, Chu J, et al. A protein interaction network for pluripotency of embryonic stem cells. Nature 2006; 444:364368.

39 Tada M, Takahama Y, Abe K, et al. Nuclear reprogramming of somatic cells by in vitro hybridization with ES cells. Curr Biol 2001; 11:1553-1558.

40 Campbell KHS, McWhir J, Ritchie WA, Wilmut I. Sheep cloned by nuclear transfer from a cultured cell line. Nature 1996; 380:6466.

41 Silva J, Chambers I, Pollard S, Smith A. Nanog promotes transfer of pluripotency after cell fusion. Nature 2006; 441:997-1001.

42 Shi W, Wang H, Pan G, et al. Regulation of the pluripotency marker Rex-1 by Nanog and Sox2. J Biol Chem 2006; 281:2331923325.

43 Zhang J, Wang X, Chen B, et al. Expression of Nanog gene promotes NIH3T3 cell proliferation. Biochem Biophys Res Commun 2005; 338:1098-1102.

44 Takahashi K, Yamanaka S. Induction of pluripotent stem cells from mouse embryonic and adult fibroblast cultures by defined factors. Cell 2006; 126:663-676. 